\title{
Mobile Free Space Optical Communication System
}

\author{
Xian Wang ${ }^{\mathrm{a}}$, Chi Yeh Hsu ${ }^{\mathrm{b}}$, and Xiaomin Jin ${ }^{\mathrm{a}}$ \\ ${ }^{a}$ Electrical Engineering Department, 1 Grand Avenue, \\ California Polytechnic State University, San Luis Obispo, CA 93407, USA \\ ${ }^{\mathrm{b}}$ Mechanical Engineering Department, 1 Grand Avenue, \\ California Polytechnic State University, San Luis Obispo, CA 93407, USA
}

\begin{abstract}
The paper discusses a low power consumption, light weight and low cost $10 \mathrm{MHz}$ free-space optical communication (FSO). The system is designed for mounting on mobile platforms, with effective range of at least 50 meters. LightEmitting Diodes (LEDs) and Laser diodes (LDs) are used in the transmitter for comparison for their relative performance in the system. The receiver features a beam splitter that feeds part of the data link optical signal to a quadrant photodetector and controls a tracking/stabilization system using microcontroller. Since almost all commercial FSO systems are mounted on fixed positions such as buildings, the paper explores the possibility and challenges of designing a FSO system for a mobile system such as remotely operated-vehicles or between ships.
\end{abstract}

Keywords: Free space, optical communication, mobile communication

\section{INTRODUCTION}

Free Space Optical Communication (FSO) offers highly directional, high bandwidth communication channels between two devices. Its links can provide fiber-like data rates over short distances with low probability of interception [1-2]. It also provides last mile solutions in many of today static communication system substituting wired or microwave system [3-5]. Recently, satellite system has begun using FSO as its communication channel thus employs a mobile system [6-7]. However, there are currently very little development on cheap, short range, mobile FSO system, for use between ships, ground vehicles and aircrafts [8]. It means the system has to be cheaper, less massive and less fragile than its counter parts in satellite systems. It is proposed to use Vertical Cavity Emitting Lasers (VCSELs) transmitters to design a low the cost of FSO system [9-10]. In our paper, we also investigate difference light source for the FSO system to reduce the system cost and make it mobile.

The mobile FSO system has it roots in fixed systems, particularly those that employ auto-tracking system [11-12]. Since many companies are involved in FSO development and production, many different design type exits. For example, the Canon's Canobeam series used laser diodes (LDs) while Solectek's FSO series uses Light-Emitting Diodes (LEDs). The Canobeam uses laser diodes with $785 \mathrm{~nm}$ wavelength and optical power output between $7 \mathrm{~mW}$ and $11 \mathrm{~mW}$ to achieve transmission distances from 100 to 1000 meters [13-14]. Canon offers their models with base-10 to base-1000 Ethernet, corresponding with bandwidth of $10 \mathrm{Mbps}$ to $1.25 \mathrm{Gbps}$. It weighs about $8 \mathrm{~kg}$ and consumes approximately 20 watts of power. Both systems are relatively bulky and they both require either AC power source or relatively high voltage DC source, which is acceptable for the fixed- mounted platforms, but for mobile platforms these requirements are impractical. The Canobeam FSO system also contains an auto tracking system that allows 2.4 degrees of free movements in vertical and horizontal directions to combat building sway. This can be used as conceptual bases for mobile systems.

In FSO systems, lasers and LEDs as the first two choices for the optical link design. Both sources have their own pros and cons. The LED system typically requires more drive current and emits less optical energy. However, compared to the laser system, LEDs are stable in various temperature and age conditions. Since our aim is to create a low cost, low power and light-weighted FSO system with auto tracking features that can be eventually mounted on a mobile platform for the last mile telecommunication. Being confined on a mobile platform, the system can be only powered by the batteries. And the power consumption of the system must be lower enough so that it will actually be practical. As the 
platform is mobile, it needs a much more robust tracking system than those used in fixed system to combat high-rise buildings sway such as Canobeam. The focus of the paper will be on the transmitter and receiver design of this system. The sensor used in the tracking system is a quadrant diode; this means that transmitted beam needs to be small enough to be tracked by the sensor. Thus the usage of the collimated laser beam is required instead of a divergent LED light source. Compared to other low cost systems such as those developed by Twilight Labs [1], our approach of using simple electronics and using of Complex Programmable Logic Device (CPLD) for the digital circuit decreases the size and power consumption of the system as well as reducing cost. In our paper, we evaluate the merits of both laser diode and LED systems. The total system design is presented in section 2. In section 3, experimental results are shown and the comparison for the each system suitability is discussed. Finally, the conclusion is presented in section 4. Our data shows that LED light is not suitable for this tracking system and the mobile system.

\section{EXPERIMENTAL SETUP}

We developed a $10 \mathrm{MHz}$ free-space optical communication with low power, light weight and low cost. This system is designed with the goal of mounting on mobile platforms, with effective range of 50 meters, with full tracking and stabilization system using servos. Since the system is in a confined space, the battery power has to be used as its power source. This puts a limited on power consumption and weight of our system. Our communication module design consumes approximately $600 \mathrm{~mW}$ of total power and outputs $15 \mathrm{~mW}$ of optical power, which consumes relative less power compared to the existing systems described above. The receiver features have a beam splitter that feeds the part of the optical data link signal to a quadrant photodetector in order to control the tracking and stabilization system. A microcontroller is used for servo controls based on the sensor information of the quadrant diodes. Since the signal is weakened by the beam splitter, we need to replace it with a more sensitivity thus more expensive photodiode or suffer a penalty on the maximum range. Since the 50 meter range considered in this system is relatively short, a cheap photodiode is used. Our system achieves a $10 \mathrm{Mbps}$ of digital bandwidth. However the bandwidth is scalable without escalation in power consumption and weight, but development cost will be substantially higher for the scope of this project, but this option remains for future development. Both LED and Laser devices are designed, measured, and compared for their performance in the optical free space communication system.

The proposed mobile free space optical communication system is shown in Fig. 1. It is setup as a full duplex optical connection between two computer systems. The computer connects to the optical system via base-10 Ethernet; the optical system then encodes the digital data into an optical beam. At the receiver end, a beam splitter is used to split the beam into two, one beam enters the communication system that coverts the optical data back into electrical data, and the other beam enters optical position detector to control the tracking system. As shown in Fig. 2 and Fig 3, since the optical beam is used for the tracking system as well as the communication system for the signal integrity purposes, a constant light beam must exist between two systems. Thus when data is present on the Ethernet line, the data will be allowed to the transmitter, otherwise a $1 \mathrm{MHz}$ signal integrity signal integrity signal will be sent. On the receiving end, the $1 \mathrm{MHz}$ signal will be filtered out. Also for the purpose of this experiment, laser diode and LED light sources are compared. Fig. 4 is the block diagram of the transmitter and the receiver design. The transmitter consists of a differential amplifier that amplifies the signal from the digital line drivers, and then passes into a wave shaper which improves rise time. A current amplifier creates enough current to drive the laser and LED diodes. The receiver consists of a reverse biased photodiode that feeds into a differentiator of the pre-amplifier instead of a Schmitt trigger for wave shaping.

The preliminary design consists of a two-axis system to be placed on both the receiver and transmitter, as both systems are placed side by side in the same package. A servo motor that drives the base allowing for 360 degrees rotational tracking adjustment, and another servo motor that controls the tilt with $+/-30$ degrees. This system is controlled by a quadrant photodetector in order to keep the cost down. In more robust systems, a true $\mathrm{X}-\mathrm{Y}$ position sensing photodetector is recommended. The sensor information is then fed into a microcontroller that controls the servo motors to keep the receiver and transmitter aligned. Since the transmitter and receiver are facing the same direction, the tracking system is only necessary on the receiver end. Aligning the receiver of unit B to the transmitter of unit A will automatically place the transmitter of unit B toward the receiver of unit A. Since both rotation and tilt adjust are present on both units, it should account for all reasonable relative movements. For the system used in range of 1 meter to 50

meters, an adjustable lens system is not necessary. In more robust systems, an auto focusing lens similar to those used in cameras could be used. The tacking system is built and integrated with the communication system. The entire system 
with transmitter, receiver, microcontroller and tracking system, is expected to consume less than 10 watts of power and weight less than 2 pounds, which is far less power and less weigh than the current FSO systems such as Canobeam and other commercial FSO systems [13-14]. The use of servos means that moving portions of the system must weigh as little as possible to enhance mechanical performance. Thus only the final stages of the receiver and transmitter as well as the beam splitter are present on the tracking housing, with all other systems mounted below. To reduce the project cost, the testing system only has one tracking unit, while the other unit is manually adjusted.

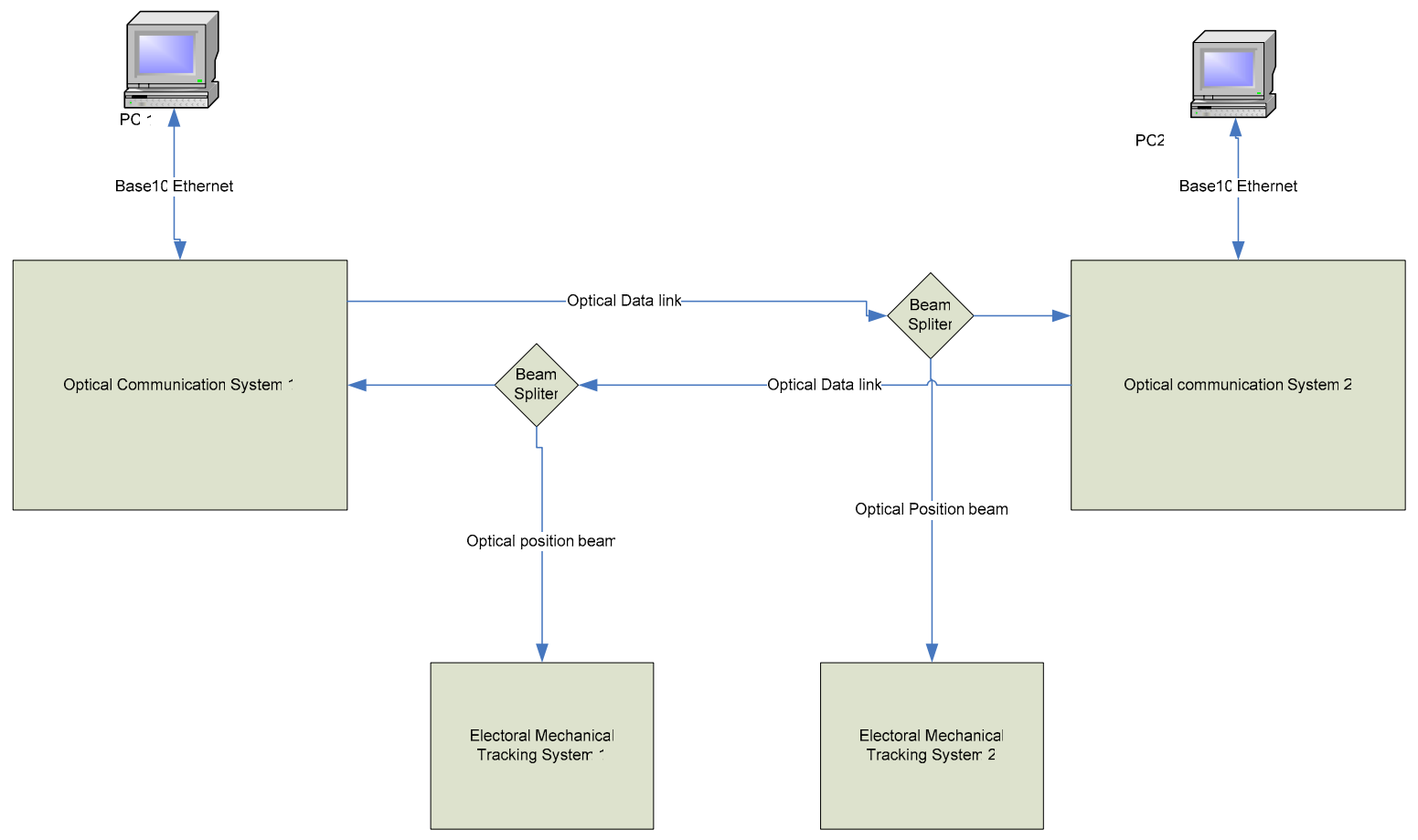

Fig. 1. The over system is setup as a full duplex optical connection between two computer systems.

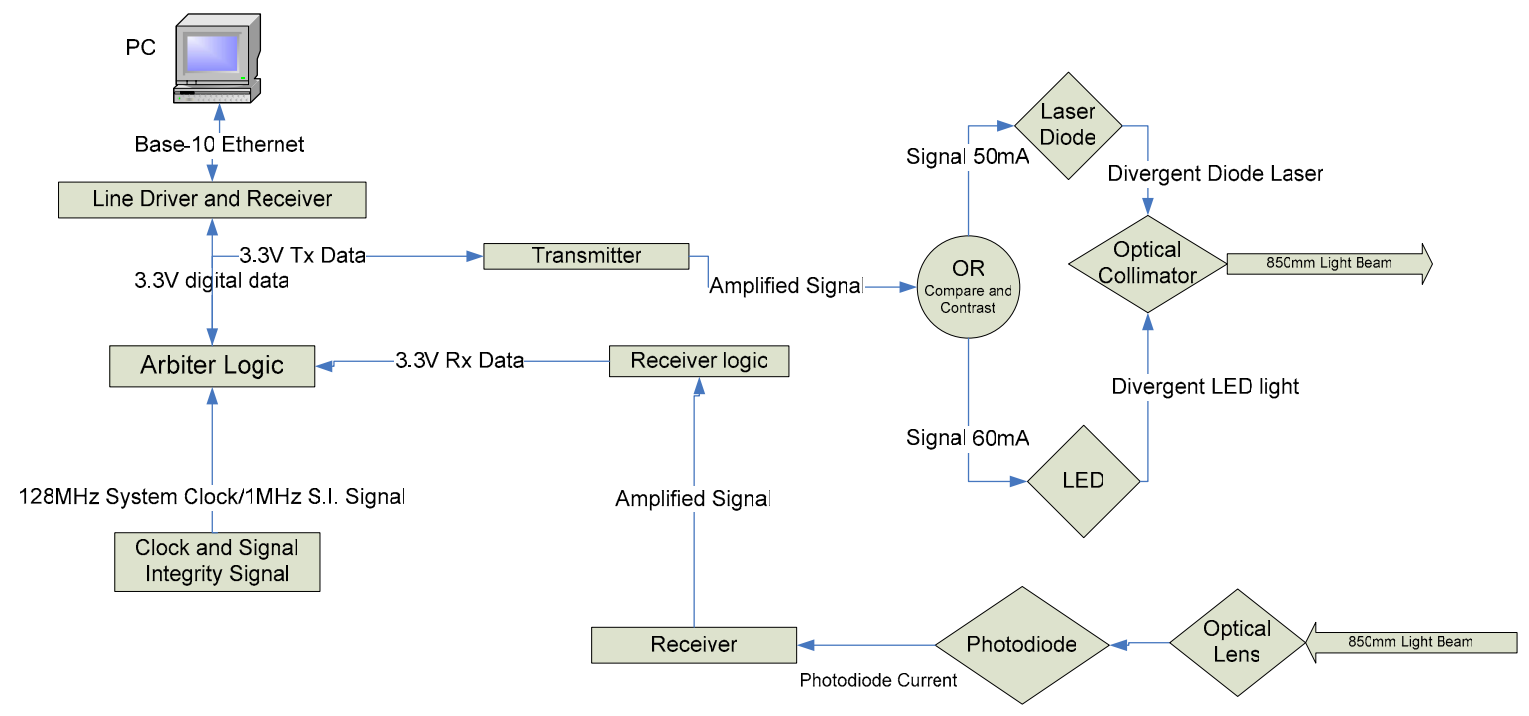

Fig. 2. Block diagram of the communication system 


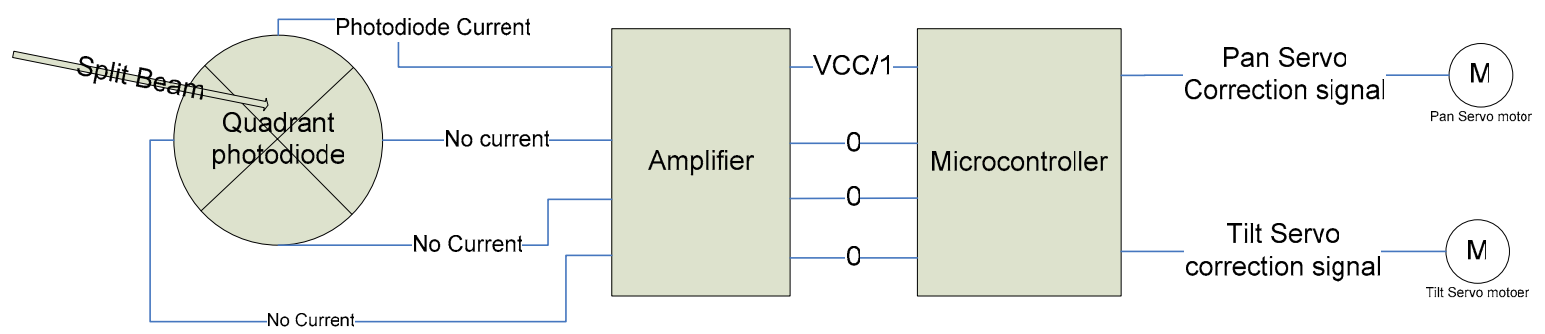

Fig 3. Block diagram of the tracking system

Transmitter

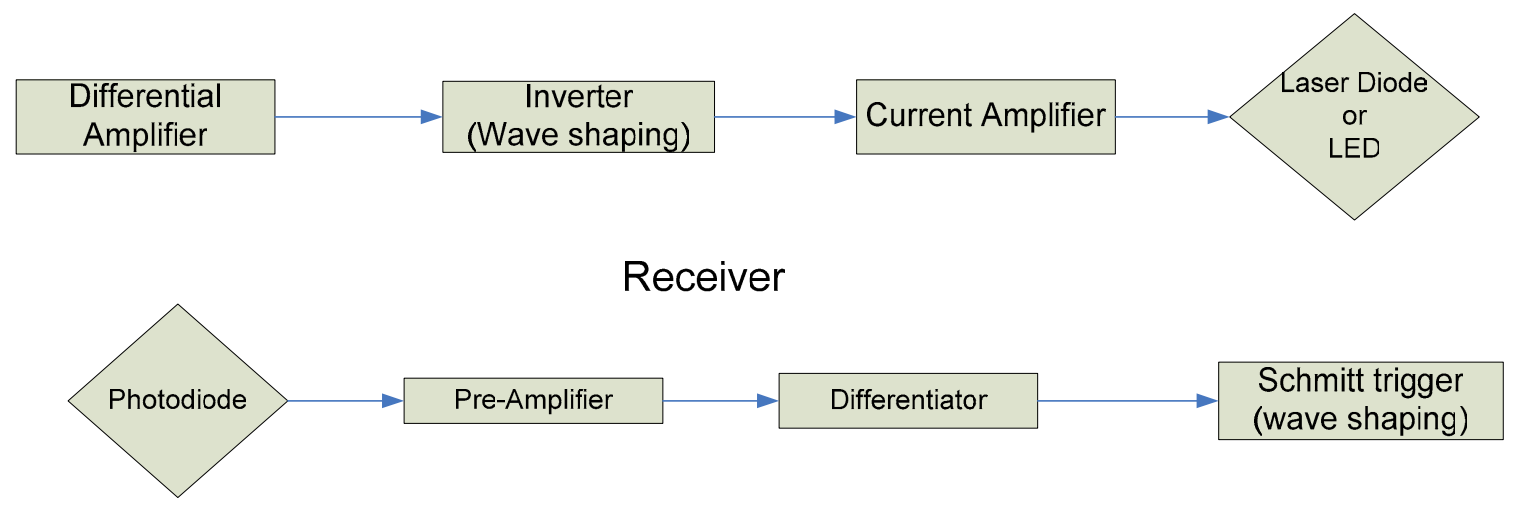

Fig. 4. The transmitter and receiver design for FSO system.

\section{RESULTS AND DISCUSSION}

The available test area for the project is limited at 50 meters. Therefore it limits the maximum range for the data collection. For the duration of the entire range waveform recovered at our digital interface stage and differentiator stagnation remained very much the same near maximum amplitudes, while the preamp stage amplitude is different. Thus that data is collected to reflect system performance. The LED beam become extremely divergent after 10 meters as it can not be detected by the IR card, however, the receiver system can still detect the waveform transmitted at about 10 meters. At distances greater than that it become very difficult to locate the beam and detect data. Optics used for a LED flashlight is used for the LED beam system, which is not as delicate and expensive as the collimator used in the laser diode system. However, this experiment shows that due to its beam divergence, the LED light source can not be used for the mobile FSO device, if a beams liter/quadrant diode method is used. Fig 5 shows our analogy link performance (a) function generator output; (b) receiver's output at 10m for Laser Diode (c) receiver's output at $50 \mathrm{~m}$ for Laser Diode; and (d) receiver's output at $10 \mathrm{~m}$ for LED. The maximum distance for LED is only $10 \mathrm{~m}$ for our experiment. In the scenario where laser diode is used as the light source, the beam remained confined even at 50 meters if the collimator is adjusted correctly. Thus makes it more suitable for beams splitter/quadrant diode method for beam tracking. The preamp output amplitude shows a decreasing trend over distance, this is a function of both atmospheric absorption and beam divergence. A way to adjust the collimator at longer distances would partially eliminate the beam divergence factor. Also the preamp output shows a triangle like waveform output, this is caused by the capacitor used to block DC current to the differentiator, and this is eliminated by the Schmitt trigger. The final performance of the signal versus distance is shown in Fig. 6 for LD system. The data clear indicate the 50m transmitter capability of our mobile FSO system, which is limited by the testing space. 


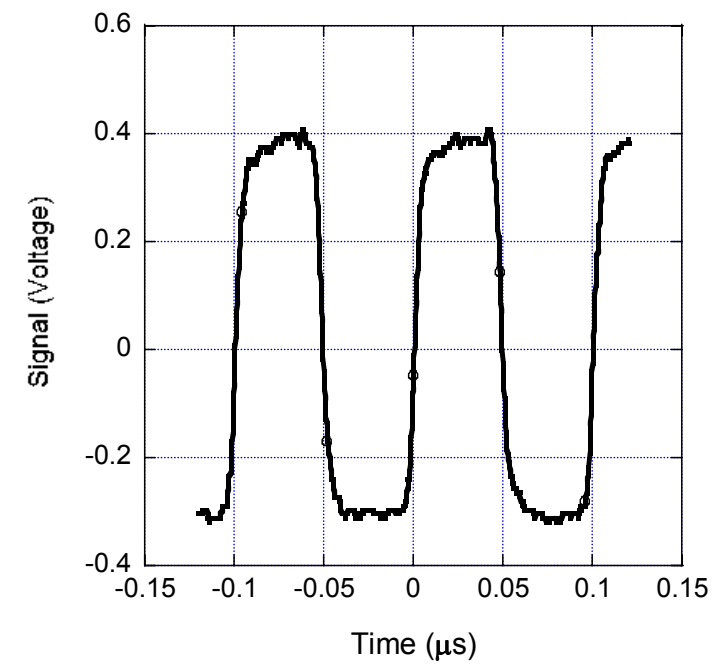

(a)

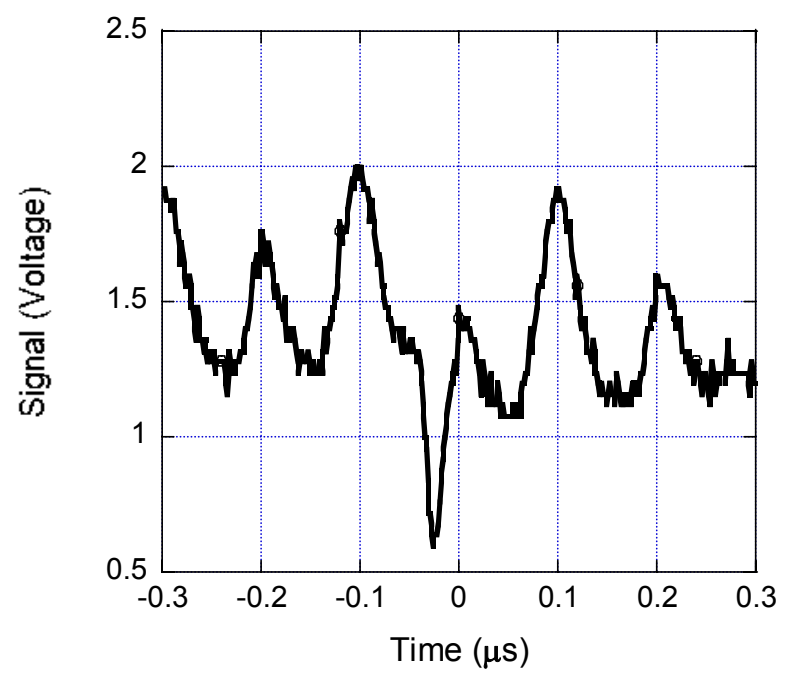

(c)

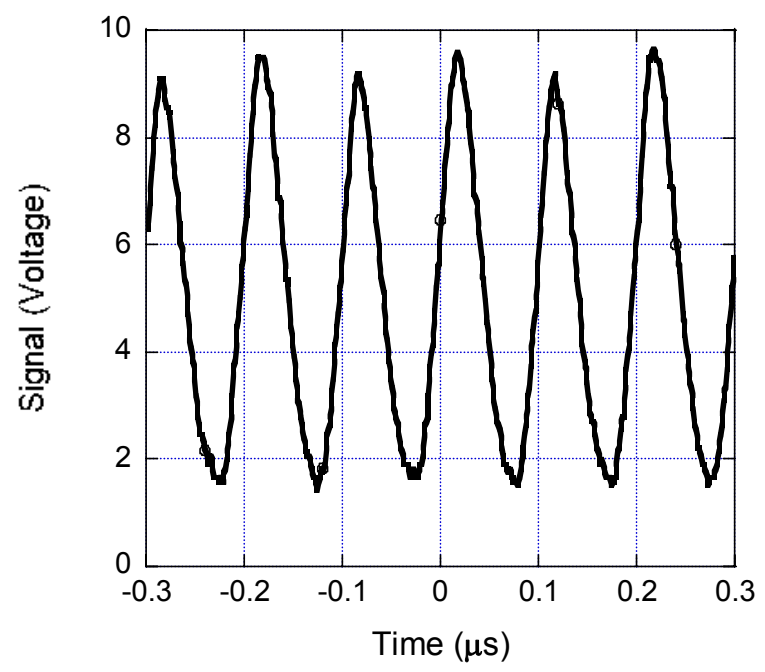

(b)

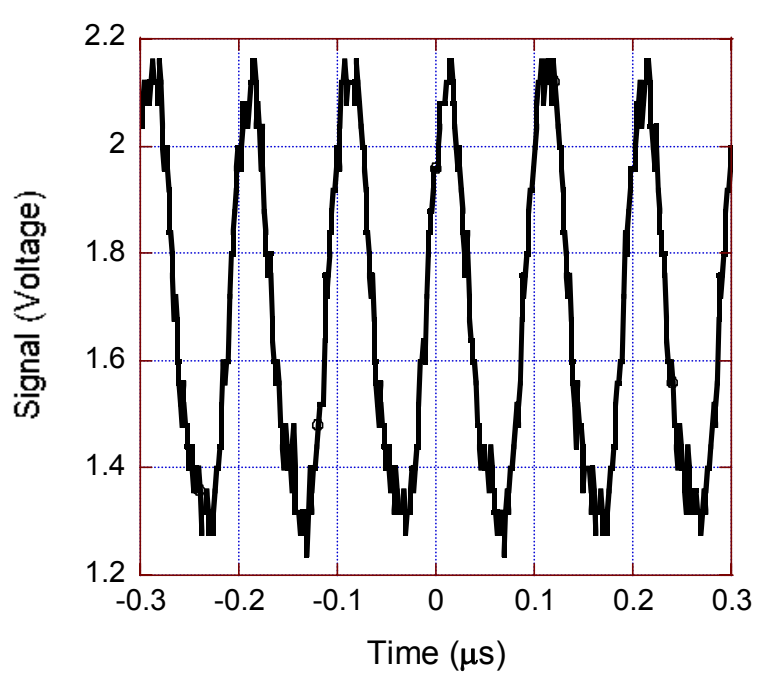

(d)

Fig. 5 Analogy link performance (a) function generator output; (b) receiver's output at 10m for Laser Diode (c) receiver's output at $50 \mathrm{~m}$ for Laser Diode; and (d) receiver's output at 10m for LED 


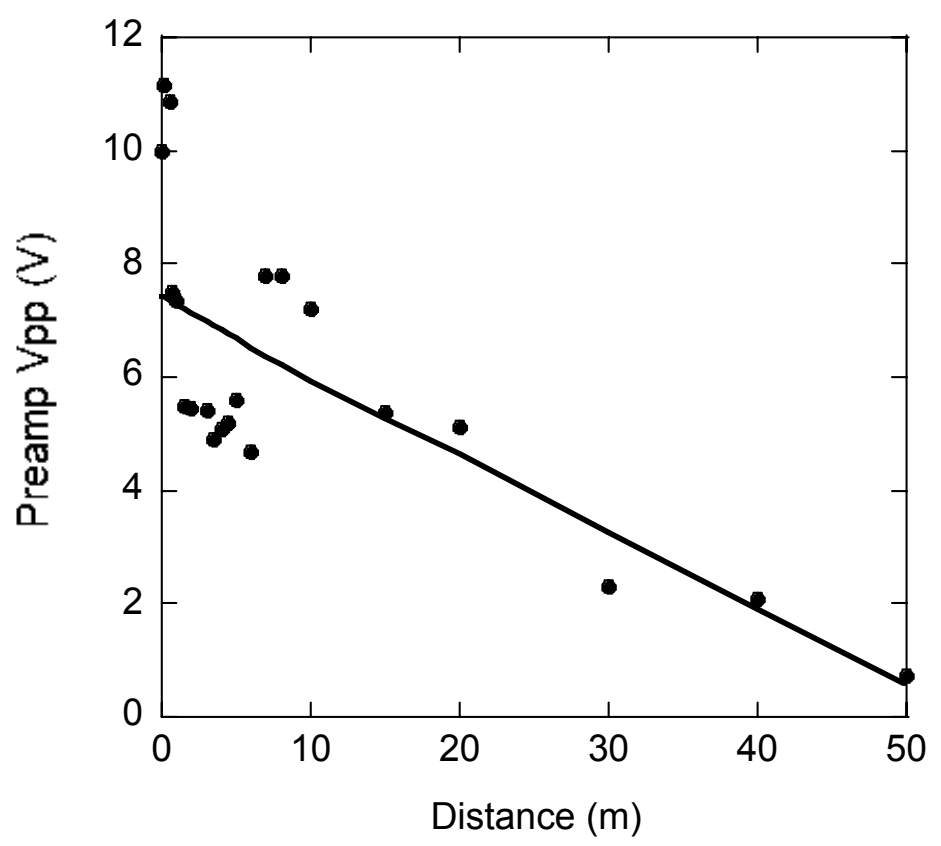

Fig 6 Signal Amplitude output versus distance for laser diode system

\section{CONCLUSION}

Since almost commercial free-space optical systems are mounted on fixed positions such as buildings, we explore the possibility and challenges of designing a mobile FSO system for remotely operated vehicles. Therefore low power and light weight are essential qualities for the mobile system. The paper demonstrates a $10 \mathrm{MHz}$ mobile FSO communication with low power consumption, light weight and low cost. The system is mounted on a mobile platform with the battery power. The effective range of 50 meters is achieved. LDs and LEDs are used respectively in the transmitter design and be compared on the system performance. The LD transmitter consumes $600 \mathrm{~mW}$ of total power, and outputs $15 \mathrm{~mW}$ of optical power, which is better than that of the LED based system.

\section{REFERENCES}

${ }^{1}$ L. Brandon, D Wilkerson, Dirk Giggenbach, and Bernhard Epple, "Concepts for fast acquisition in optical communications systems" Proceedings of the SPIE Free-Space Laser Communications VI, (2006).

2 J.M Kahn, "Secure free-space optical communication between moving platforms," The 15th Annual Meeting of the IEEE Lasers and Electro-Optics Society (LEOS) 2002, 3, 455 - 456 (2002).

3 D.L Begley, "Free-space laser communications: a historical perspective", The 15th Annual Meeting of the IEEE Lasers and Electro-Optics Society (LEOS) 2002, 3, 391-392 (2002).

${ }^{4}$ Phanumas Khumsat, Noppadol Wattanapisit, Karel Kulhavey "Optical Front-Ends for Low-Cost Laser-Based 10Mbps" Proceedings of Asia Pacific Conference on Circuits and Systems 2006, 1911 -1914 (2006)

5 Jayasri Akella, Murat Yuksel, Shiv Kalyanaraman, "Multi-channel Communication in Free-Space Optical Networks for the Last-mile" 15th IEEE Workshop on Local \& Metropolitan Area Networks (LANMAN) 2007, 43-48 ( 2007)

6 J.C. Juarez, A. Dwivedi, A.R. Mammons, S.D. Jones, V. Weerackody, R.A. Nichols, "Free-Space Optical Communications for Next-generation Military Networks", IEEE Communications Magazine, 44, 46 - 51 (2006).

7 H. Hemmati, "Status of free-space optical communications program at JPL", 2000 IEEE Aerospace Conference Proceedings, 3, 101 - 105 (2000). 
8 M. Karatay, M.S. Dinleyici, "Low-cost free-space optical communication system design", Proceedings of the IEEE $12^{\text {th }}$ Signal Processing and Communications Applications Conference 2004, 700 - 703 (2004).

9 M. Yoshikawa, A. Murakami, J. Sakurai, H. Nakayama, T. Nakamura, "High power VCSEL devices for free space optical communications", Proceedings of the $55^{\text {th }}$ Electronic Components and Technology Conference 2005, 2, 1353 1358 (2005).

${ }^{10}$ J.R. Minch, D.R. Gervais, D.J. Townsend, "Adaptive Transceivers for Mobile Free-Space Optical Communications", Military Communications Conference (MILCOM) 2006, 1 - 5, (2006).

${ }^{11}$ Tom Garlington, MAJ Joel Babbitt, and George Long "Analysis of Free Space Optics as a Transmission Technology" Army Information System Engineering Command, (2005).

${ }^{12}$ V. W. S. Chan, "Free-Space Optical Communications", Journal of Lightwave Technology, 24(12), 4750 - 4762 (2006).

${ }^{13}$ Canon corp., Canobeam DT100, http://www.usa.canon.com/html/industrial_canobeam/canobeam/canobeam100.html.

${ }^{14}$ Solectk Corp, FSO-100E, http://www.solectek.com/products.php?prod=fso\&page=feat.

${ }^{15}$ Tianping Deng, Yimin Lu, and Xiaoxiao, "Performance evaluation of free space optical communication system", Proceedings of International Conference on Wireless Communications, Networking and Mobile Computing, 2005, 1, $587-590(2005)$.

${ }^{16}$ Tariq Manzur, "Free Space Optical Communications (FSO)", 2007 IEEE Avionics, Fiber-Optics and Photonics Technology Conference, 21 - 21 (2007). 